\title{
Complete heart block caused by cardiac echinococcosis and successfully treated with albendazole
}

\author{
Murat Özdemir, Erdem Diker, Sinan Aydoḡdu, Siber Göksel
}

A 40 year old man who had hydatid cysts in his liver and right kidney was found to have complete heart block during preoperative evaluation and was referred to our clinic. Blood pressure was $120 / 80 \mathrm{~mm} \mathrm{Hg}$ and the heart rate was $40 / \mathrm{min}$ and regular. The physical examination was normal except for hepatomegaly which reached almost $15 \mathrm{~cm}$ at the midclavicular line. The electrocardiogram showed complete atrioventricular block with a ventricular rate between 35 and 40 beats per minute. He had a white cell count of $12100 / \mathrm{mm}^{3}$ and $13 \cdot 2 \%$ eosinophilia on the peripheral blood smear. The erythrocyte sedimentation rate was $48 \mathrm{~mm} / \mathrm{h}$. A haemagglutination test for hydatid disease was positive (titre 1/1600). Abdominal ultrasonography revealed hydatid cysts in the liver and the right kidney. A cross sectional echocardiogram showed a $19 \times 19$ mm cystic mass on the membranous portion of the IVS, protruding to the left ventricular outflow tract but creating no pressure gradient on Doppler echocardiography (figure A).

These findings led to the patient being treated with albendazole $200 \mathrm{mg}$ twice a day. After four weeks of chemotherapy he underwent laparotomy under transient transvenous pacing. Right nephrectomy together with drainage of the hepatic cyst was performed. $\mathrm{He}$ had an uneventful postoperative course. Complete heart block persisted for three weeks after laparotomy then it converted to normal sinus rhythm with left bundle branch block. The follow up echocardiogram showed that the cardiac cyst had become smaller.

The patient refused to undergo cardiac surgery. He was given a total of five cycles of chemotherapy each of which comprised 28 days of albendazole $200 \mathrm{mg}$ twice a day and 14 days drug free. No side effects occurred throughout the course of therapy. Normal sinus rhythm with left bundle branch block continued; complete heart block never reappeared. The echocardiographic examination performed when the course of treatment was complete showed that the cystic cavity had completely disappeared, only an area of hyperechogenicity with some calcification was present (figure B). No periods of complete heart block were evident on 24 hour Holter monitoring.

Cardiac echinococcosis is an uncommon disease. Involvement of the interventricular septum has been reported in $2-9 \%$ of cases. ${ }^{1}$ Ventricular septal cysts may interfere with conduction pathways, produce obstructive symptoms, or cause tricuspid regurgitation. A cardiac hydatid cyst may spontaneously die and calcify, but it can lead to pericardial tamponade or anaphylactic shock if it ruptures into the pericardial sac or the ventricular cavity.

Cross sectional echocardiography is the best diagnostic procedure for cardiac hydatid disease. Eosinophilia is present in $20 \%$ of cases. The indirect haemagglutination test has a sensitivity of $82 \%$ and a specificity of $91 \%$ for the diagnosis of hydatid disease.

Owing to the high incidence of sudden death $(20 \%)$ and rupture into the cardiac chambers $(39 \%)$, or into the pericardium $(10 \%)$, it is generally accepted that cardiac hydatid disease must be treated surgically unless the disease is recurrent or inoperable. ${ }^{1}$

Albendazole is the most promising agent for the treatment of echinococcosis at present. In a series of 253 patients with echinococcosis in various organs $72(28.5 \%)$ were regarded as cured, $129(51 \%)$ as improved, $46(18 \cdot 1 \%)$ as unchanged, and $6(2.4 \%)$ worse with albendazole therapy. ${ }^{2}$ In only $10.6 \%$ of patients given albendazole preoperatively were viable hydatid cysts shown in the surgical specimen. The recommended standard therapeutic regimen is 4 or 5 cycles of 30 days $(10 \mathrm{mg} / \mathrm{kg} /$ day $)$ and a rest period of two weeks between the cycles. ${ }^{1}$ Frequent monitoring of hepatic, renal, and bone marrow function is mandatory during treatment.

Most of the cysticidal activity of albendazole seems to occur within the first 2-3 months of treatment and if the cyst is not smaller after two months' treatment, further treatment is unlikely to help. ${ }^{3}$ When the echinococcus cyst dies, the outer wall often becomes calcified. The material in the lumen degenerates into a thick amorphous paste which no longer contains viable germinal elements. It has been suggested that cysts that show structural disorganisation (separation of membranes, loss of definition, lamination, increase in ultrasonographic density, and calcification) are not viable and may in time resolve. ${ }^{2}$

We know of published reports of two cases of cardiac echinococcosis causing complete atrioventricular block ${ }^{2}$ and both were success- 


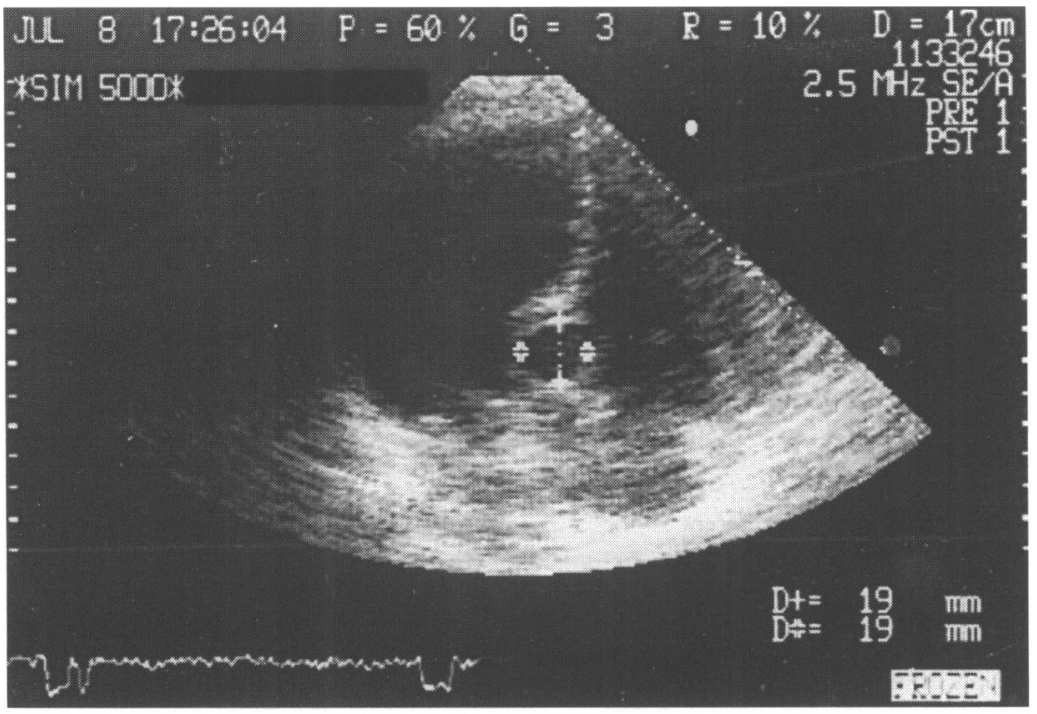

$A$

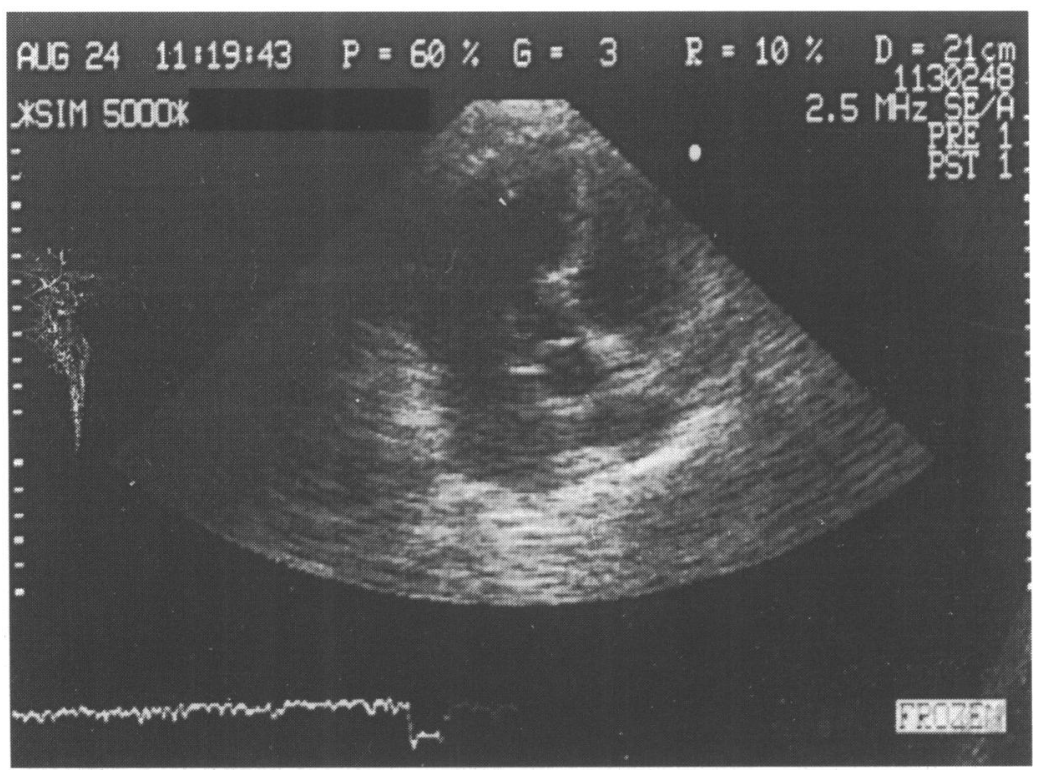

$B$

(A) Before albendazole treatment there was a $19 \times 19 \mathrm{~mm}$ cystic mass on the membraneous septum protruding to the left ventricular outflow tract (echocardiographic apical four chamber view). (B) After albendazole treatment the cystic cavity had disappeared. Only an area of hyperechogenity and some calcification remained (echocardiographic apical four chamber view). fully treated by surgery. ${ }^{4}$ In our case, albendazole treatment resulted in conversion of complete heart block to sinus rhythm with left bundle branch block. Moreover, the echocardiographic findings (obliteration of the cystic cavity, increase in the ultrasound density, and calcification) achieved after albendazole treatment suggest that the cyst had been killed.

1 Lanzoni AM, Barrios V, Moya JL, Epeldegui A, Celemin D, Lafuente C, Asin-Cardiel E. Dynamic left ventricular outflow obstruction caused by cardiac echinococcosis. Am Heart F 1992;124:1083-5.

2 Horton RJ. Chemotherapy of echinococcus infection in man with albendazole. Trans $R$ Soc Trop Med Hyg 1989; 83:97-102.

3 Aggarwal P, Wali JP. Albendazole in the treatment of pulmonary echinococcosis. Thorax 1991;46:599-600.

4 Ottino G, Villani M, De Paulis R, Trucco G, Viara A. Restoration of atrioventricular conduction after surgical removal of a hydatid cyst of the interventricular septum. $f$ Thorac Cardiovasc Surg 1987;93:144-7.

5 Gavrilescu S, Gavrilescu M, Streian C, Luca C. Complete atrioventricular block due to cardiac echinococcosis. Cardiology 1979;64:215-21. 